Pedro Vladimir Gonzalez Castellanos

\title{
Modelamento de Efeitos de Multipercurso Atmosférico em Enlaces Microondas em Visibilidade
}

Dissertação de Mestrado

DEPARTAMENTO DE ENGENHARIA ELÉTRICA

Programa de Pós-Graduação em Engenharia Elétrica 


\section{Pontifícia Universidade $\mathrm{C}_{\text {atólica }}$

Pedro Vladimir Gonzalez Castellanos

Modelamento de Efeitos de Multipercurso Atmosférico em Enlaces Microondas em Visibilidade

Dissertação de Mestrado

Dissertação apresentada como requisito parcial para obtenção do título de Mestre pelo Programa de PósGraduação em Engenharia Elétrica da PUC-Rio.

Orientador: Luiz Alencar da Silva Mello 


\section{Pontifícia Universidade Católica $_{\text {a }}$ \\ DO RIO DE JANEIRO}

Pedro Vladimir Gonzalez Castellanos

\section{Modelamento de Efeitos de Multipercurso Atmosférico em Enlaces Microondas em Visibilidade}

Dissertação apresentada como requisito parcial para obtenção do título de Mestre pelo Programa de PósGraduação em Engenharia Elétrica da PUC-Rio. Aprovada pela Comissão Examinadora abaixo assinada.

Luiz Alencar da Silva Mello Orientador Centro de Estudos em Telecomunicações - PUC-Rio

Prof. Erasmus Couto Brasil de Miranda UCP

Prof. Gláucio Lima Siqueira Centro de Estudos em Telecomunicações - PUC-Rio

Prof. Marco Antonio Grivet Mattoso Maia Centro de Estudos em Telecomunicações - PUC-Rio

Prof. Ney Augusto Dumont Coordenador(a) Setorial do Centro Técnico Científico - PUC-Rio 
Todos os direitos reservados. É proibida a reprodução total ou parcial do trabalho sem autorização da universidade, do autor e do orientador.

\section{Pedro Vladimir Gonzalez Castellanos}

Graduou-se em Engenharia de Sistemas na Universidad Francisco de Paula Santander (Cúcuta - Norte de Santander Colômbia em julho de 2001).

Ficha Catalográfica

Gonzalez Castellanos, Pedro Vladimir

Modelamento de efeitos de multipercurso atmosférico em enlaces microondas em visibilidade / Pedro Vladimir Gonzalez Castellanos; orientador: Luiz Alencar da Silva Mello. - Rio de Janeiro : PUC, Departamento de Engenharia Elétrica, 2003.

86 f. : il. ; $30 \mathrm{~cm}$

Dissertação (mestrado) - Pontifícia Universidade Católica do Rio de Janeiro, Departamento de Engenharia Elétrica.

Incluí referências bibliográficas.

1. Engenharia elétrica - Teses. 2. Desvanecimento por multipercurso. 3. Duração de eventos de desvanecimento. I. Mello, Luiz Alencar da Silva. II. Pontifícia Universidade Católica do Rio de Janeiro. Departamento de Engenharia Elétrica. III. Título. 
A Deus Todo Poderoso e

À Virgem da Torcoroma

A minha Família 


\section{Agradecimentos}

Ao professor Luiz Alencar da Silva Mello, pela orientação, os ensinamentos, pela compreensão e parceria na realização deste trabalho.

A PUC-Rio pelos auxílios concedidos e a oportunidade. Aos professores das diferentes matérias cursadas pelos ensinamentos e a Rodolfo Sabóia pela ajuda.

A minha família, a meus pais Blanca Lilia e Francisco Antonio, aos meus irmãos em especial ao meu irmão Jaime e a Julia pelo apoio e compreensão nos momentos difíceis, e a minha Natacha quem me acompanhou no final deste projeto.

Aos amigos Luis, Jose Alberto, Tatiana pelo apoio na parte inicial do mestrado.Aos amigos e colegas do PAA Godinho, Marcela, Luciana, Ana Cristina, e Pedro Pablo, pelo companheirismo.

Aos funcionários do CETUC, pelo carinho e atenção em especial a Maria Luzia. 


\section{Resumo}

Gonzalez Castellanos, Pedro Vladimir. Modelamento de efeitos de multipercurso atmosférico em enlaces microondas em visibilidade. Rio de Janeiro, 2003. 86p. Dissertação de Mestrado - Departamento de Engenharia Elétrica, Pontifícia Universidade Católica do Rio de Janeiro.

Os desvanecimentos por multipercurso atmosférico são a principal causa de degradação do desempenho de enlaces rádio digitais de alta capacidade, acentuando os efeitos do ruído térmico e da interferência intersimbólica na taxa de bits errados. A previsão da distribuição cumulativa de probabilidades destes desvanecimentos em função de parâmetros do enlace e características rádio climáticas da região é de fundamental importância no projeto de sistemas rádio digitais terrestres.

Existem na literatura técnica métodos para previsão do desvanecimento em enlaces individuais, mas não para um tronco de microondas formado por vários enlaces. Medidas realizadas no Brasil indicam que a usual suposição de decorrelação total entre desvanecimentos profundos em enlaces consecutivos em um mesmo tronco é pessimista, podendo levar a uma indisponibilidade combinada maior do que a real.

Neste trabalho foram analisados dados experimentais de 15 enlaces na região centro-oeste do Brasil e 5 enlaces no Japão e desenvolvido um modelo empírico de previsão da distribuição conjunta do desvanecimento por multipercurso atmosférico conjunta em enlaces adjacentes. $O$ modelo apresenta boa concordância com os resultados experimentais e fornece uma nova expressão para o cálculo da indisponibilidade em troncos de microondas formados por vários enlaces.

Os dados experimentais foram utilizados ainda para modelar a duração de eventos de atenuação por multipercurso em enlaces em visibilidade. Mostrou-se que a distribuição de duração de eventos é bem caracterizada por distribuições de Weibull. Esta caracterização permite a distinção entre eventos de perda de qualidade (duração inferior a 10 segundos) e de indisponibilidade (duração superior a 10 segundos).

\section{Palavras-chave}

Desvanecimentos por multipercurso, Desvanecimento conjunto em enlaces consecutivos, Duração de eventos de desvanecimento. 


\section{Abstract}

Gonzalez Castellanos, Pedro Vladimir. Modeling atmospheric multipath fading in Line-of-sight microwave links. Rio de Janeiro, 2003. 86p. MSc. Dissertation - Electrical Engineering Department, Pontifícia Universidade Católica do Rio de Janeiro.

Multipath fading is the main cause of performance degradation in high capacity line-of-sight microwave links, increasing enhancing the effects of the thermal noise and intersymbol interference in the bit error rate.

Tested methods are available in the literature for the prediction of cumulative probability distribution multipath fading in individual link but not in a microwave network formed by several tandem links. Measurements carried out in Brazil indicate that the usual assumption that deep fade events in consecutive links are totally uncorrelated may be pessimistic for tropical regions and may lead to the prediction excessive multihop links unavailability.

Experimental data obtained in measurements of multipath fading in 15 pairs of tandem links located in the center-western region of Brazil were analyzed to provide cumulative distributions of attenuation in each pair. Similar results are available in the literature for 5 pairs of links in Japan.

Based on these results, an empirical model was developed for the prediction of the joint probability distribution the multipath fading in adjacent links was developed. The attenuation simultaneously exceeded in both links for given percentage of time can be predicted as a function of the equiprobable values of attenuation exceeded in the individual links, path lengths and operation frequency.

The predicted distributions show good agreement with the experimental results and provide an expression for the calculation of unavailability of line-ofsight tandem links.

The experimental data had been used still to model the duration of events of attenuation for multipath of links in visibility. One revealed that the distribution of duration of events well is characterized by distributions of Weibull. This characterization allows the distinction between events of loss of quality (lowest duration at 10 seconds) and of non-availability (higher duration the 10 seconds).

\section{Keywords}

Multipath fading, Simultaneous fading in multihops links, Duration events of attenuation. 


\section{Sumário}

1 Introdução 14

2 Conceitos Básicos $\quad 17$

2.1. Perda em Espaço Livre 17

2.2. Propagação Sobre Terra Plana 19

2.3. Efeitos da atmosfera na propagação 20

2.3.1. Refratividade da atmosfera 21

2.3.2. Gradiente da refratividade $\quad 22$

2.3.3. Efeitos refrativos na curvatura do raio - o fator $\mathrm{K}$

2.4. Efeitos da Difração $\quad 27$

2.5. Reflexões no Solo 28

2.6. Desvanecimento em enlaces rádio 28

2.6.1. Desvanecimento por multipercurso atmosférico 29

2.6.2. Desvanecimentos planos 30

2.7. Modelos de previsão da distribuição de probabilidades dos desvanecimentos por multipercurso 31

2.7.1. Modelo de previsão do ITU-R (Recomendação P.530-7) 32

2.7.2. Modelo de ITU-R (Recomendação P.530-10) 35

2.8. Modelo do ITU-R para enlaces em tronco 37

2.9. Duração de Desvanecimentos 38

3 Descrição do experimento e resultados das medidas 39

3.1. Sistema de Medidas 43

3.2. Sistema de aquisição e análise de dados 4

3.2.1. Unidade de Aquisição de Dados 44

3.2.2. Programas de pré-processamento de dados 45

3.2.3. Edição de séries temporais 45

3.2.4. Programa de análise estatística de dados 46

3.2.5. Resultados experimentais 47

4 Previsão da atenuação em enlaces consecutivos 48

4.1. Método para previsão da atenuação conjunta 49 
4.1.1. Desenvolvimento do método empírico 49

4.1.2. Avaliação da precisão do método de previsão 52

4.2. Método de previsão com dependência micro-climática 56

4.2.1. Avaliação da precisão do método com micro-climas 59

4.3. Comparação geral dos modelos de previsão 62

5 Duração de eventos de desvanecimento 65

5.1. Modelamento da duração de eventos de desvanecimento 65

5.2. Ajuste de distribuições usando a distribuição de Weibull 68

5.3. Ajuste de distribuições usando a distribuição Normal 70

5.4. Comportamento dos parâmetros $\beta$ e $\eta$ da distribuição de Weibull 71

$\begin{array}{ll}6 \text { Conclusões e sugestões } & 75\end{array}$

$\begin{array}{ll}7 \text { Referências Bibliográficas } & 78\end{array}$

8 Anexo A - Séries Temporais da Atenuação Conjunta Para os enlaces analisados e comparação com os modelos desenvolvidos. 80 


\section{Lista de figuras}

Figura 2.1 Propagação Sobre Terra Plana 20

Figura 2.2 Valores mensais de No para o mês de fevereiro 23

Figura 2.3 Valores mensais de No para o mês de agosto 24

Figura 2.4 Horizonte geométrico e de uma onda de rádio 24

Figura 2.5 Curvatura do raio para diferentes valores de K 25

Figura 2.6 Registro do sinal recebido, serie temporal. 29

Figura 3.1 Distribuição geográfica dos locais de medida 42

Figura 3.2 Set-up Experimental de Aquisição dos dados 43

Figura 3.3 Serie temporal da potencia recebida visualizada no programa tsedit.exe 46

Figura 3.4 Exemplo de distribuições de atenuação para um par de enlaces $\quad 47$

Figura 4.1 Atenuação conjunta contra atenuação mínima entre os enlaces individuais 49

Figura 4.2 Atenuação conjunta contra distância mínima entre os enlaces individuais 50

Figura 4.3 Atenuação conjunta contra distância máxima entre os enlaces individuais $\quad 50$

Figura 4.4 Atenuação conjunta contra freqüência de operação 50

Figura 4.5 Valores de atenuação conjunta previstos contra medidos 53

Figura 4.6 Valores da distribuição de resíduos 53

Figura 4.7 Comparação do modelo com os dados medidos do site Barracão Queimado (Brasil) 55

Figura 4.8 Comparação do modelo com os dados medidos do site Santa Luzia

(Brasil) 55

Figura 4.9 Comparação do modelo com os dados medidos do site Futago (Japão) 55

Figura 4.10 Comparação do modelo com os dados medidos do site Awagatake (Japão) 56

Figura 4.11 Regiões de micro-climáticas dos enlaces 58

Figura 4.12 Valores de atenuação conjunta previstos contra medidos $\quad 59$

Figura 4.13 Valores da distribuição de resíduos 60

Figura 4.14 Comparação do modelo com os dados medidos em Itiquira $\quad 60$

Figura 4.15 Comparação do modelo com os dados medidos em Santa Luzia 61 
Figura 4.16 Comparação dos modelos com os dados medidos em Funtago

Figura 4.17 Comparação do modelo com os dados medidos em Awagatake 61

Figura 4.18 Valor médio do erro relativo 63

Figura 4.19 Desvio padrão do erro relativo 63

Figura 4.20 Desvio padrão do erro relativo 63

$\begin{array}{ll}\text { Figura 5.1 Arquivo de saída do programa temp } & 67\end{array}$

Figura 5.2 Duração de eventos dos níveis de 5 ate $20 \mathrm{~dB} \quad 67$

Figura 5.3 Duração de eventos dos níveis de 25 ate $40 \mathrm{~dB}$

Figura 5.4 Densidade de probabilidade de Weibull (linha cheia), contra os dados medidos, enlace Alto Araguaia - Alto Garças, nível de $5 \mathrm{~dB} \quad 69$

Figura 5.5 Densidade de probabilidade de Weibull (linha cheia), contra os dados medidos, enlace Cacoal - Alto Garças, nível $10 \mathrm{~dB} \quad 69$

Figura 5.6 Densidade de probabilidade de Weibull (linha cheia), contra os dados medidos, enlace Barracão Queimado-Nova Oeste, nível 15 dB 69

Figura 5.7 Média, desvio padrão e valor RMS do erro relativo do ajuste da densidade de probabilidades da duração de eventos pela distribuição de $\begin{array}{ll}\text { Weibull } & 70\end{array}$

Figura 5.8 Média, desvio padrão e valor RMS do erro relativo do ajuste da densidade de probabilidades da duração de eventos pela distribuição $\begin{array}{ll}\text { Normal } & 71\end{array}$

Figura 5.9 Parâmetro $\beta$ contra o nível de atenuação e a distância 72

Figura 5.10 Parâmetro $\beta$ contra a distância e a freqüência 72

Figura 5.11 Parâmetro $\beta$ contra a freqüência e o nível 72

Figura 5.12 Parâmetro $\beta$ contra a inclinação e o nível 73

Figura 5.13 Parâmetro $\eta$ contra o nível e a distancia 73

Figura 5.14 Parâmetro n contra a distância e a freqüência $\quad 74$

Figura 5.15 Parâmetro n contra a freqüência e o nível 74

Figura 5.16 Parâmetro $\eta$ contra a inclinação e o nível $\quad 74$

Figura A.1 Comparação do modelo com os dados medidos em Futago 80

Figura A.2 Comparação do modelo com os dados medidos em Yanbara $\quad 80$

Figura A.3 Comparação do modelo com os dados medidos em Awagatake 81

Figura A.4 Comparação do modelo com os dados medidos em Tsukude 81

Figura A.5 Comparação do modelo com os dados medidos em Santa Luzia 81

Figura A.6 Comparação do modelo com os dados medidos em ingleses 82

Figura A.7 Comparação do modelo com os dados medidos em Itiquira 82

Figura A.8 Comparação do modelo com os dados medidos em Rondonópolis 82 
Figura A.9 Comparação do modelo com os dados medidos em Araguaia 83

Figura A.10 Comparação do modelo com os dados medidos em Mineiros 83

Figura A.11 Comparação do modelo com os dados medidos em Jataí 83

Figura A.12 Comparação do modelo com os dados medidos em Livramento 84

Figura A.13 Comparação do modelo com os dados medidos em Fza Sta Luzia 84

Figura A.14 Comparação do modelo com os dados medidos em B. Queimado 84

Figura A.15 Comparação do modelo com os dados medidos em Iracema 85

Figura A.16 Comparação do modelo com os dados medidos em Cacoal 85

Figura A.17 Comparação do modelo com os dados medidos em Jaru 85

Figura A.18 Comparação do modelo com os dados medidos em São Pedro 86 


\section{Lista de tabelas}

Tabela 2.1 Valores do coeficiente $C_{0}$ para as três faixas de alturas da antena mais baixa e os três tipos de terreno 33

Tabela 3.1 Número de enlaces por freqüência da portadora 40

Tabela 3.2 Dados dos enlaces que foram analisados 41

Tabela 4.1 Erro Relativo da Media e o desvio padrão 54

Tabela 4.2 Valores do parâmetro rádio climático 57

Tabela 4.3 Média, desvio padrão e valor RMS do erro percentual 59

Tabela 4.4 Valor médio do erro relativo 62

Tabela 4.5 Desvio padrão do erro relativo 62

Tabela 4.6 Valor RMS do erro relativo 62 